\title{
List coloring hypergraphs
}

\author{
Penny Haxell* \\ Department of Combinatorics and Optimization \\ University of Waterloo \\ Waterloo, Ontario, Canada \\ pehaxell@uwaterloo.ca
}

\author{
Jacques Verstraete ${ }^{\dagger}$ \\ Department of Mathematics \\ University of California \\ San Diego, CA \\ jverstra@math.ucsd.edu
}

Submitted: Apr 29, 2010; Accepted: Sep 6, 2010; Published: Sep 22, 2010

Mathematics Subject Classification: 05C15, 05C65

\begin{abstract}
Let $H$ be a hypergraph and let $L_{v}: v \in V(H)$ be sets; we refer to these sets as lists and their elements as colors. A list coloring of $H$ is an assignment of a color from $L_{v}$ to each $v \in V(H)$ in such a way that every edge of $H$ contains a pair of vertices of different colors. The hypergraph $H$ is $k$-list-colorable if it has a list coloring from any collection of lists of size $k$. The list chromatic number of $H$ is the minimum $k$ such that $H$ is $k$-list-colorable. In this paper we prove that every $d$-regular three-uniform linear hypergraph has list chromatic number at least $\left(\frac{\log d}{5 \log \log d}\right)^{1 / 2}$ provided $d$ is large enough. On the other hand there exist $d$-regular three-uniform linear hypergraphs with list chromatic number at $\operatorname{most} \log _{3} d+3$. This leaves the question open as to the existence of such hypergraphs with list chromatic number $o(\log d)$ as $d \rightarrow \infty$.
\end{abstract}

\section{Introduction}

A hypergraph $H$ is $k$-uniform if every edge of $H$ has size $k$, and $d$-regular if every vertex of $H$ is in exactly $d$ edges of $H$. A hypergraph is linear if any pair of distinct edges of the hypergraph intersect in at most one vertex. Let $H$ be a hypergraph and let $L_{v}: v \in V(H)$ be sets; we refer to these sets as lists. A list coloring of $H$ is an assignment of an element of $L_{v}$ to each $v \in V(H)$ in such a way that every edge of $H$ contains a pair of vertices assigned different elements. The hypergraph $H$ is called $k$-list-colorable if it has a list coloring from any collection of lists of size $k$. The list chromatic number $\chi_{\ell}(H)$ of $H$, also called the choice number of $H$, is the minimum $k$ such that $H$ is $k$-list-colorable. In this paper, we study the list chromatic number of linear uniform regular hypergraphs.

*Partially supported by NSERC

${ }^{\dagger}$ Research supported by an Alfred P. Sloan Research Fellowship and NSF Grant DMS-0800704 


\subsection{List coloring graphs}

The notion of list-coloring is a generalization of the notion of proper coloring, and has been studied extensively for graphs. In particular, Alon [1] showed that every bipartite graph of minimum degree at least $d$ has list chromatic number at least $\frac{1}{2} \log _{2} d$, improving

a preceding lower bound of order $\frac{\log d}{\log \log d}$ as $d \rightarrow \infty$ in [2]. On the other hand it is known [9] that the complete bipartite graph $K_{d, d}$ with $d$ vertices in each part satisfies $\chi\left(K_{d, d}\right)=$ $(1+o(1)) \log _{2} d$. It has been asked whether every bipartite graph with maximum degree $d$ has list chromatic number $O(\log d)$ (see Alon and Krivelevich [4]) but this tantalizing problem remains open. Here and in what follows, the logarithm is taken to be the natural logarithm unless a base is explicitly displayed.

\subsection{List coloring hypergraphs}

There seem to be very few results on list colorings of hypergraphs. Perhaps the most famous question on colorings of hypergraphs is the Erdős-Faber-Lovász conjecture (see [6]): given an $n$-uniform linear hypergraph consisting of $n$ edges, there is vertex-coloring of the hypergraph for which every edge receives all $n$ colors. Equivalently, the conjecture states that any graph comprising the union of $n$ edge-disjoint cliques of size $n$ has chromatic number $n$. Via a more general result on coloring linear hypergraphs, Kahn [11] showed that that an $n$-uniform linear hypergraph can be vertex-colored with $n+o(n)$ colors in such a way that the vertices in each edge all receive different colors. Recently, a simple proof of the Erdős-Faber-Lovász conjecture was announced. For Steiner triple systems with $n$ vertices - three-uniform hypergraphs in which every pair of vertices is covered exactly once - a lower bound of order $\log n / \log \log n$ for the list chromatic number was shown in [10]. In this paper, we concentrate on giving bounds on the list chromatic number of all linear regular three-uniform hypergraphs, which we refer to as triple systems. We shall check in this paper that the complete $r$-partite $r$-uniform hypergraph $K_{r \times n}$ with parts of size $n$ has list chromatic number asymptotic to $\log _{r} n$ as $n \rightarrow \infty$.

\subsection{Main Theorem}

The main result of this paper shows that every $d$-regular linear triple system has large list chromatic number:

Theorem 1 There exists a constant $d_{0}$ such that for $d \geqslant d_{0}$, every d-regular linear triple system $H$ has

$$
\chi_{\ell}(H)>\left(\frac{\log d}{5 \log \log d}\right)^{1 / 2} .
$$

On the other hand, there exists a d-regular linear triple system with list chromatic number at most $\log _{3} d+3$.

The requirement of linearity in this theorem is necessary. Consider the triple system consisting of vertex set $V=V_{1} \cup \cdots \cup V_{n} \cup W$ with $\left|V_{i}\right|=2$ and $|W|=n$ and where 
the edge set $E$ consists of $\left\{e \subset V:|e \cap W|=1, \exists i: V_{i} \subset e\right\}$. This is an $n$-regular triple system whose list chromatic number is two, since for any assignment of lists we can choose different colors for the two vertices inside each $V_{i}$.

Relative to the theorem above, the most relevant open question is the existence of $d$-regular linear triple systems with list chromatic number $o(\log d)$ as $d \rightarrow \infty$. We leave this as an open problem.

Problem. Do there exist three-uniform $d$-regular linear hypergraphs of list chromatic number $o(\log d)$ as $d \rightarrow \infty$ ?

\section{List coloring complete $r$-partite hypergraphs}

In this section, we show that for infinitely many $d$, there exists a linear $d$-regular triple system with list chromatic number at $\operatorname{most} \log _{3} d+3$. The example is the following threepartite triple system $K_{3 \times d}(L)$ : if $L$ is any $d \times d$ latin square, then we create a linear triple system with three parts as follows. We index the rows of $L$ by a set $R$ of size $d$, the columns by a set $C$ of size $d$, and then let $R, C,[d]$ denote the parts of $K_{3 \times d}(L)$. Then the edges of $K_{3 \times d}(L)$ are all triples $\{r, c, i\}$ with $r \in R, c \in C$, and such that $i$ is in position $(r, c)$ in $L$. Note that by definition of a latin square there are no repeated entries in any row or column of $L$, so the triple system $K_{3 \times d}(L)$ is $d$-regular and linear. We will show that it has list chromatic number at most $\log _{3} d+3$ by showing that the complete $r$-partite hypergraph $K_{r \times d}$ - which contains $K_{3 \times d}(L)$ when $r=3$ - has list chromatic number at most $\log _{r} d+3$. We will also show that $K_{r \times d}$ has list chromatic number at least $\log _{r} d-O(\log \log d)$ as $d \rightarrow \infty$. Our argument is based on that of [9]. We define a hypergraph $H$ to be $r$-colorable if the list assignment in which all lists are $\{1, \ldots, r\}$ admits a list coloring of $H$. The following lemma will be used:

Lemma 2 Let $m_{r}(k)$ denote the minimum number of edges in a $k$-uniform hypergraph which is not $r$-colorable. Then as $k \rightarrow \infty$,

$$
r^{k-1} \leqslant m_{r}(k) \leqslant O_{r}\left(k^{2} r^{k}\right) .
$$

Proof $\triangleright$ A random $r$-coloring of a hypergraph consists in choosing uniformly at random a color from $[r]$ independently for each vertex in the hypergraph. The lower bound of $r^{k-1}$ in the lemma follows from the fact that in a random $r$-coloring of a $k$-uniform hypergraph with $m$ edges, the expected number of edges whose vertices are all assigned the same color is $r^{1-k} m$, so if $m<r^{k-1}$, then the hypergraph is $r$-colorable. In fact, this is all we shall need to prove $\chi_{\ell}\left(K_{r \times n}\right) \leqslant \log _{r} n+3$. The upper bound on $m_{r}(k)$ also follows from probabilistic methods; we sketch the proof for $r>2$, since it is very similar to the proof for $r=2$ given in [7] (see also Alon and Spencer [5], Page 9). We consider a hypergraph $H$ with vertex set $\left[r k^{2}\right]$ constructed by randomly and uniformly selecting sets of size $k$ in $T$ independent rounds from $\left[r k^{2}\right]$. We shall take

$$
T=\left\lceil 2 k^{2}(e r)^{k} \log r\right\rceil .
$$


An $r$-coloring of $H$ is a partition $\left(X_{1}, X_{2}, \ldots, X_{r}\right)$ of the vertex set $\left[r k^{2}\right]$ of $H$. An edge of $H$ is monochromatic if it lies entirely in some $X_{i}$. We say that an $r$-coloring of $H$ fails in round $i$ if in the $i$ th round, the chosen set of size $k$ is monochromatic. The aim is to show that the expected number of $r$-colorings which do not fail in any round is less than one, and we follow the computation of Erdős [7]. The probability that the edge chosen at round $i$ is monochromatic for a given coloring $\left(X_{1}, X_{2}, \ldots, X_{r}\right)$ is exactly

$$
\left(\begin{array}{c}
r k^{2} \\
k
\end{array}\right)^{-1} \sum_{i=1}^{r}\left(\begin{array}{c}
\left|X_{i}\right| \\
k
\end{array}\right) .
$$

By convexity of binomial coefficients, the sum is a minimum when $\left|X_{1}\right|=\left|X_{2}\right|=\cdots=$ $\left|X_{r}\right|=k^{2}$ :

$$
\sum_{i=1}^{r}\left(\begin{array}{c}
\left|X_{i}\right| \\
k
\end{array}\right) \geqslant r\left(\begin{array}{c}
k^{2} \\
k
\end{array}\right) .
$$

Using the standard bounds $\left(\begin{array}{c}r k^{2} \\ k\end{array}\right) \leqslant(e r k)^{k}$ and $\left(\begin{array}{c}k^{2} \\ k\end{array}\right) \geqslant k^{k}$, the probability that an edge chosen at round $i$ is monochromatic is at least $r /(e r)^{k}$. It follows that the probability that a given coloring $\left(X_{1}, X_{2}, \ldots, X_{r}\right)$ does not fail at any round is at most

$$
\left(1-\frac{r}{(e r)^{k}}\right)^{T}
$$

since the rounds are independent. Now $T$ was chosen so that this quantity is less than $r^{-r k^{2}}$. Since the number of $r$-colorings of $\left[r k^{2}\right]$ is $r^{r k^{2}}$, we conclude that the expected number of $r$-colorings which do not fail at any round is less than one. In particular, there is a witness $H$ to this event. This hypergraph $H$ has exactly $T$ edges and is not $r$-colorable, as required.

We use the bounds on $m_{r}(k)$ in the above lemma to give bounds on $\chi_{\ell}\left(K_{r \times m}\right)$ as follows:

Theorem 3 For all $r \geqslant 2$ and as $m \rightarrow \infty$,

$$
\chi_{\ell}\left(K_{r \times m}\right)=(1+o(1)) \log _{r} m .
$$

Proof $\triangleright$ If we can show $\chi_{\ell}\left(K_{r \times m}\right) \leqslant k$ when $r m=m_{r}(k)-1$ and $\chi_{\ell}\left(K_{r \times m}\right) \geqslant k+1$ when $m=m_{r}(k)$, then we are done using the bounds in the last lemma, since $r m+1 \geqslant r^{k-1}$ implies $k \leqslant \log _{r} m+3$ and $m \leqslant C k^{2} r^{k}$ implies $k \geqslant \log _{r} m-2 \log _{r} \log _{r} m-O(1)$ when $m \rightarrow \infty$, as required. First we show that for $m=m_{r}(k)$, we have $\chi_{\ell}\left(K_{r \times m}\right)>k$. By definition of $m_{r}(k)=m$, there is a $k$-uniform hypergraph $F$ with $m$ edges such that $F$ is not $r$-colorable. We let the lists in the $i$ th part $V_{i}$ of $K_{r \times m}$ be exactly the edges of $F$, for $1 \leqslant i \leqslant r$. We claim there is no coloring of $K_{r \times m}$ from this list assignment. Suppose, for a contradiction, that there is a list coloring of $K_{r \times m}$ from these lists, and let $S_{1}, S_{2}, \ldots, S_{r}$ be the sets of colors used on the vertices of $V_{1}, V_{2}, \ldots, V_{r}$, respectively. Note that each $S_{i}$ is actually a set of vertices of $F$. We observe that

$$
\bigcap_{i=1}^{r} S_{i}=\emptyset
$$


otherwise a certain color appears on a vertex $v_{i} \in V_{i}$ for $1 \leqslant i \leqslant r$, in which case $\left\{v_{1}, v_{2}, \ldots, v_{r}\right\}$ is a monochromatic edge of $K_{r \times m}$. Since each $S_{i}$ is a transversal of the edges in $F, T_{i}=V(F) \backslash S_{i}$ does not contain any edges of $F$ for $1 \leqslant i \leqslant r$. Since $\bigcap S_{i}=\emptyset$, every vertex of $F$ is in the complement of some $S_{i}$ and therefore $T_{1} \cup \cdots \cup T_{r}=V(F)$. This contradicts that $F$ is not $r$-colorable, and hence $\chi_{\ell}\left(K_{r, m}\right)>k$ when $m=m_{r}(k)$.

Conversely we show $\chi_{\ell}\left(K_{r \times m}\right) \leqslant k$ when $r m=m_{r}(k)-1$. Consider an assignment of lists of size $k$ to the $r m$ vertices of $K_{r \times m}$, and let $F$ be the $k$-uniform hypergraph of all those lists. Then $F$ has an $r$-coloring since $|F|<m_{r}(k)$. Fix an $r$-coloring of $F$, say $V(F)=T_{1} \cup T_{2} \cup \cdots \cup T_{r}$. The list $L_{v}$ at a vertex $v \in V_{i}$ must contain an element not in $T_{i}$, otherwise $L_{v}$ would be monochromatic under the coloring $T_{1} \cup T_{2} \cup \cdots \cup T_{r}$ of $F$. To each $v \in V_{i}$ we assign an arbitrary element of $L_{v} \backslash T_{i}$, for $1 \leqslant i \leqslant r$. We claim this is a proper coloring of $K_{r \times m}$. If not, then there is an edge $\left\{v_{1}, v_{2}, \ldots, v_{r}\right\}$ in $K_{r \times m}$ where $v_{i} \in V_{i}$, and such that every $v_{i}: 1 \leqslant i \leqslant r$ receives the same color, which is an element of $T_{j}$ for some $j \in[r]$. However, $v_{j}$ was assigned an element of $L_{v_{j}} \backslash T_{j}$, which is a contradiction. Therefore $\chi_{\ell}\left(K_{r \times m}\right) \leqslant k$. This completes the proof.

It appears to be an interesting question to determine $f(d)=\min _{L} \chi_{\ell}\left(K_{3 \times d}(L)\right)$ where the minimum is over all $d \times d$ latin squares. In particular, it would be interesting to determine whether $f(d)=o(\log d)$ as $d \rightarrow \infty$.

\section{Lemmas}

The probabilistic lemmas we use to prove Theorem 1 are given here. The first lemma is the Chernoff bound, one of the basic tools in probabilistic methods - see for example Alon and Spencer [5].

Lemma 4 Let $Z_{1}, Z_{2}, \ldots, Z_{n}$ be identically distributed independent random variables where $P\left(Z_{i}=1\right)=p$ and $P\left(Z_{i}=0\right)=1-p$, and let $S$ be their sum. Then for any $\epsilon \in(0,1]$,

$$
P(|S-E(S)|>\epsilon E(S)) \leqslant 2 \exp \left(-\epsilon^{2} E(S) / 2\right) .
$$

We also require the Lovász Local Lemma [8] in the following form. Here and in what follows, $A^{c}$ denotes the complement of an event $A$.

Lemma 5 Let $A_{1}, A_{2}, \ldots, A_{n}$ be events in a probability space, such that each $A_{i}$ is mutually independent of any subset of events indexed by the set $[n] \backslash J_{i}$ for some dependency set $J_{i} \subset[n]$ where $\max \left|J_{i}\right|=\triangle$. Suppose that

$$
P\left(A_{i}\right) \leqslant \frac{1}{4 \triangle}
$$

for all $i \in[n]$. Then

$$
P\left(\bigcap_{i=1}^{n} A_{i}^{c}\right) \geqslant \exp \left(-\frac{n}{\triangle}\right) .
$$




\section{Proof of Theorem 1}

We are given a $d$-regular linear triple system $H$, and we want to come up with a collection of lists on the vertices from which no coloring is possible and where the lists are as large as possible. The idea of the proof is to show that if we assign random lists of length $s$ in $[t]:=\{1,2, \ldots, t\}$ to the vertices of $H$, for some carefully chosen values of $s$ and $t$, then with positive probability there is no proper coloring of $H$ from these lists. We will choose $s=(\log d)^{1 / 2}(5 \log \log d)^{-1 / 2}$ and $t=(8 s)^{s(s+4)}$ and put $p:=1 /(8 s)^{3} t$. If $d$ is a large enough constant, then it is straightforward to check that the parameters $p, s, t$ satisfy (for completeness a verification is written in the appendix):

$$
\begin{aligned}
t \exp \left(-s^{2} p^{3} d / 4 t^{2}\right) & <1 / 64 d^{2} \\
\exp \left(-n /(8 s)^{2} t\right) & <s^{-3 p n} \\
\exp \left(-p^{2} n / 8\right) & <\exp \left(-n / d^{2}\right)
\end{aligned}
$$

For any $K \leqslant(8 s)^{s}$, we record that standard bounds on binomial coefficients (see Appendix : Lemma 6) give

$$
\left(\begin{array}{c}
t / K \\
s
\end{array}\right)>(2 K)^{-s}\left(\begin{array}{l}
t \\
s
\end{array}\right)
$$

\subsection{Preprocessing}

Define random sets $X \subset V(H)$ and $Y \subset V(H)$ by independently placing each vertex of $V(H)$ into $X$ with probability $p^{2}$ and into $Y$ with probability $p$ and into $Z=V(H) \backslash(X \cup Y)$ with probability $1-p-p^{2}$. Let $H^{\prime}$ denote the three-partite hypergraph consisting of all $\{x, y, z\} \in H$ such that $x \in X, y \in Y$ and $z \in Z$. We write $d(x, y)>2$ to denote that in $H^{\prime}$ every edge on $x$ is disjoint from every edge on $y$ - in other words $x$ and $y$ are at distance more than two. For $y \in Y$ let $\Gamma_{X}(y)=\left\{x \in X:\{x, y, z\} \in H^{\prime}\right.$ for some $\left.z\right\}$ and for $z \in Z$ let $\Gamma_{X Y}(z)=\left\{(x, y) \in X \times Y:\{x, y, z\} \in H^{\prime}\right\}$. Define $d_{X}(y)=\left|\Gamma_{X}(y)\right|$ and $d_{X Y}(z)=\left|\Gamma_{X Y}(z)\right|$. Since $H$ is a $d$-regular linear hypergraph, we have for $y \in Y$ and $z \in Z$,

$$
E\left(d_{X}(y)\right)=2 p^{2}\left(1-p-p^{2}\right) d \quad \text { and } \quad E\left(d_{X Y}(z)\right)=2 p^{3} d .
$$

Let $A_{y}: y \in Y$ be the events $p^{2} d<d_{X}(y)<4 p^{2} d$ and let $A_{z}: z \in Z$ be the event $p^{3} d<d_{X Y}(z)<4 p^{3} d$.

Claim 1. With positive probability, each event $A_{X}=\left\{\frac{1}{2} p^{2} n<|X|<2 p^{2} n\right\}$ and $A_{Y}=\left\{\frac{1}{2} p n<|Y|<2 p n\right\}$ and every $A_{y}: y \in Y$ and every $A_{z}: z \in Z$ occurs.

Proof. We shall apply the Chernoff Bound with $\epsilon=1 / \sqrt{8}$. The event $A_{y}$ contains the event $\left|d_{X}(y)-E\left(d_{X}(y)\right)\right|<\epsilon E\left(d_{X}(y)\right)$, since $p<1 / 8^{3}$, and clearly $A_{z}$ contains the event 
$\left|d_{X Y}(z)-E\left(d_{X Y}(z)\right)\right|<\epsilon E\left(d_{X Y}(z)\right)$. Since vertices are placed independently in the sets $X$ and $Y$, Lemma 4 shows

$$
P\left(A_{y}^{c}\right) \leqslant 2 \exp \left(-\frac{p^{2} d}{8}\right) \quad \text { and } \quad P\left(A_{z}^{c}\right) \leqslant 2 \exp \left(-\frac{p^{3} d}{8}\right)
$$

and similarly $P\left(A_{X}^{c}\right) \leqslant 2 \exp \left(-p^{2} n / 8\right)$ and $P\left(A_{y}^{c}\right) \leqslant 2 \exp (-p n / 8)$. A dependency graph of the events $A_{y}: y \in Y$ and $A_{z}: z \in Z$ has maximum degree at most $\triangle=4 d^{2}$, since any single event $A_{v}^{c}$ is mutually independent of any set of events $A_{w}^{c}: d(v, w)>2$. By (1), both the bounds in (5) are easily less than 1/4 $\triangle$. By Lemma 5, with probability at least $\exp \left(-n / 4 d^{2}\right)$ every $A_{y}: y \in Y$ and every $A_{z}: z \in Z$ occurs. By $(3), \exp \left(-n / 4 d^{2}\right)>$ $P\left(A_{X}^{c} \cup A_{Y}^{c}\right)$, and so with positive probability, the events $A_{X}$ and $A_{Y}$ and every $A_{y}: y \in Y$ and $A_{z}: z \in Z$ occurs. This proves the claim.

For the remainder of the proof, we work in a subhypergraph $H^{\prime}$ for which all the events in Claim 1 hold, and we assume that $X, Y$ and $Z$ are the parts of $H^{\prime}$.

\subsection{Choice of lists in $X$}

First we assign lists to $X$. We choose uniformly and independently random lists of size $s$ from $[t]$ for the vertices of $X$. For $y \in Y$, let $B_{y}$ be the event that no color appears in more than $2 s d_{X}(y) / t$ lists in $\Gamma_{X}(y)$ and for $z \in Z$ let $B_{z}$ be the event that no color appears in more than $2 s d_{X Y}(z) / t$ lists in

$$
\Gamma_{X}(z):=\bigcup\left\{x \in X: \exists y \in Y,(x, y) \in \Gamma_{X Y}(z)\right\}
$$

Claim 2. With positive probability, every $B_{y}: y \in Y$ and every $B_{z}: z \in Z$ occurs.

Proof. Since $H^{\prime}$ is linear, note that $\left|\Gamma_{X}(z)\right|=d_{X Y}(z)$. The expected number of times a particular color appears in lists in $\Gamma_{X}(y)$ is exactly $s d_{X}(y) / t$ and the expected number of times a particular color appears in $\Gamma_{X}(z)$ is $s d_{X Y}(z) / t$. Since the lists are chosen independently, the probability that a particular color appears in more than $2 s d_{X}(y) / t$ lists in $\Gamma_{X}(y)$ is at most $2 \exp \left(-s d_{X}(y) / 2 t\right)$, by the Chernoff Bound with $\epsilon=1$. A similar statement holds for the colors in $\Gamma_{X}(z)$, and since there are at most $t$ colors, we deduce from the union bound that

$$
\begin{aligned}
& P\left(B_{y}^{c}\right)<2 t \exp \left(-s d_{X}(y) / 2 t\right)<2 t \exp \left(-s p^{2} d / 2 t\right) \\
& P\left(B_{z}^{c}\right)<2 t \exp \left(-s d_{X Y}(z) / 2 t\right)<2 t \exp \left(-s p^{3} d / 2 t\right) .
\end{aligned}
$$

A dependency graph of the events $B_{v}^{c}$ has maximum degree at most $\triangle=4 d^{2}$, since $B_{v}^{c}$ is mutually independent of any events $B_{w}^{c}: d(v, w)>2$. By (1), we easily have $P\left(B_{y}^{c}\right)<1 / 4 \triangle$ and $P\left(B_{z}^{c}\right)<1 / 4 \triangle$, and so Lemma 5 completes the proof of Claim 2 .

From now on we fix an assignment of lists $\mathcal{L}^{*}$ of size $s$ from $[t]$ to the vertices of $X$, such that every $B_{y}: y \in Y$ and every $B_{z}: z \in Z$ occurs. Let $\rho_{y}$ be the number of colors 
used at least $d_{X}(y) / 2 t$ times on $\Gamma_{X}(y)$. Since $B_{y}$ occurs,

$$
\left(t-\rho_{y}\right) \frac{d_{X}(y)}{2 t}+\rho_{y} \frac{2 s d_{X}(y)}{t} \geqslant d_{X}(y)
$$

and it follows that $\rho_{y}>t / 4 s$. Since every $B_{y}$ occurs, we have that for each $y \in Y$ and each coloring of $X$ from $\mathcal{L}^{*}$ there exists a set $S_{y}$ of $\lceil t / 4 s\rceil$ colors each appearing at least $p^{2} d / 2 t$ times in $\Gamma_{X}(y)$.

\subsection{Choice of lists in $Y$}

Now we independently and randomly assign lists $L_{y}$ of size $s$ from $[t]$ to the $y \in Y$. For a fixed coloring of $X$ from $\mathcal{L}^{*}$, if $L_{y} \subset S_{y}$, then any color selected from $L_{y}$ results in at least $p^{2} d / 2 t$ vertices $x \in \Gamma_{X}(y)$ of the same color as $y$. Let $B_{\chi}$ be the event that under a coloring $\chi$ of $X, L_{y} \subset S_{y}$ for at least $\frac{1}{2}(8 s)^{-s}|Y|$ vertices $y \in Y$, and let $B=\bigcap_{\chi} B_{\chi}$ where the intersection is over all colorings of $X$ from $\mathcal{L}^{*}$. For $z \in Z$, let $C_{z}$ be the event that no color appears on the list at $x$ and at $y$ for at least $4 s^{2} d_{X Y}(z) / t^{2}$ pairs $(x, y) \in \Gamma_{X Y}(z)$.

Claim 3. With positive probability, $B$ as well as every $C_{z}: z \in Z$ occurs.

Proof. We observe that for every $\chi$ and every $y$,

$$
P\left(L_{y} \subset S_{y}\right) \geqslant\left(\begin{array}{c}
t / 4 s \\
s
\end{array}\right) /\left(\begin{array}{l}
t \\
s
\end{array}\right)>(8 s)^{-s}
$$

using (4) with $K=4 s$. Therefore the expected number of events $L_{y} \subset S_{y}$ is at least $(8 s)^{-s}|Y|$. Since the $L_{y}$ are chosen independently, the Chernoff Bound with $\epsilon=1 / 2$ shows that for a fixed coloring $\chi$ of $X$,

$$
P\left(B_{\chi}^{c}\right)<2 \exp \left(-\frac{|Y|}{8(8 s)^{s}}\right)
$$

Since there are at most $s^{|X|}$ colorings of $X$, and since by Claim $1|Y| \geqslant p n / 2$ and $|X| \leqslant$ $2 p^{2} n$, the expected number of colorings $\chi$ of $X$ for which $B_{\chi}^{c}$ occurs is at most

$$
2 \exp \left(-\frac{|Y|}{8(8 s)^{s}}\right) \cdot s^{|X|}<2 \exp \left(-\frac{p n}{16(8 s)^{s}}+2 p^{2} n \log s\right)<\exp \left(-\frac{p n}{20(8 s)^{s}}\right) .
$$

By (2), with room to spare, this is less than $\exp \left(-n / d^{2}\right)$, and so Markov's Inequality gives $P\left(B^{c}\right)<\exp \left(-n / 2 d^{2}\right)$. For $x \in \Gamma_{X}(z)$, there is a unique $y \in \Gamma_{Y}(z)$ such that $(x, y) \in \Gamma_{X Y}(z)$, since $H$ is linear. The chance that $y$ is assigned a list containing a particular color in $L_{x}$ is exactly $s / t$. Fix a color $i$, and let $C_{z}^{i}$ be the event that color $i$ appears on the list at $x$ and at $y$ for at least $4 s^{2} d_{X Y}(z) / t^{2}$ pairs $(x, y) \in \Gamma_{X Y}(z)$. Recall that since every $B_{z}$ occurs, color $i$ appears at most $2 s d_{X Y}(z) / t$ times in $\Gamma_{X}(z)$. The expected number of $(x, y) \in \Gamma_{X Y}(z)$ such that $i \in L_{x} \cap L_{y}$ is at most $2 s^{2} d_{X Y}(z) / t^{2}$. Since lists are assigned independently, we may apply the Chernoff Bound with $\epsilon=1$ to obtain

$$
P\left(C_{z}^{i}\right)<2 \exp \left(-s^{2} d_{X Y}(z) / t^{2}\right) .
$$


Since there are $t$ colors in total, the union bound shows

$$
P\left(C_{z}^{c}\right)<2 t \exp \left(-s^{2} d_{X Y}(z) / t^{2}\right) .
$$

By Claim 1, this is at most

$$
t \exp \left(-s^{2} p^{3} d / 2 t^{2}\right)
$$

Now $C_{z}^{c}$ is mutually independent of any events $C_{w}^{c}: d(w, z)>2$, so a dependency graph of events $C_{z}^{c}$ has maximum degree at most $\triangle=4 d^{2}$. By $(1), P\left(C_{z}^{c}\right)<1 / 4 \triangle$ and so Lemma 5 shows that with probability at least $\exp \left(-n / 4 d^{2}\right)$, every $C_{z}$ occurs. Using the preceding bound on $P\left(B^{c}\right)$, we see that with positive probability every $C_{z}: z \in Z$ and $B$ occurs.

Let us now extend our list assignment $\mathcal{L}^{*}$ to include a list assignment of $s$ elements from $[t]$ to each $y \in Y$, such that $B$ and each $C_{z}: z \in Z$ occurs.

\subsection{Choice of lists for $Z$}

We have assigned lists $\mathcal{L}^{*}$ to the vertices of $X$ and $Y$ in such a way that in every coloring of $X$, there is a set $S \subset Y$ of size at least $p n / 4(8 s)^{s}$ with the property that for $y \in S$, $L_{y} \subset S_{y}$ (this is the event $B$ ), and for every $z \in Z$, every color appears on the list at $x$ and at $y$ for at most $4 s^{2} d_{X Y}(z) / t^{2}$ pairs $(x, y) \in \Gamma_{X Y}(z)$ (these are the events $C_{z}$ ). Now we show that there exists an assignment of lists to $Z$ from which no coloring of $H^{\prime}$ is possible.

Let $G$ denote the bipartite graph consisting of parts $X$ and $Y$ and edges $\bigcup_{z \in Z} \Gamma_{X Y}(z)$. If $\kappa$ is any fixed coloring of $X \cup Y$, then since $B$ occurs, whichever color from $L_{y}$ that is assigned to $y$ by $\kappa$ results in at least $p^{2} d / 2 t$ pairs $(x, y) \in G$ such that $x$ and $y$ have the same color. Therefore we have at least

$$
\sum_{y \in S} \frac{p^{2} d}{2 t}>\frac{p^{3} d n}{8 t(8 s)^{s}}
$$

pairs $(x, y) \in G$ such that $x$ and $y$ are assigned the same color by $\kappa$. We call these monochromatic edges of $G$. Let $T$ denote the set of $z \in Z$ such that $\Gamma_{X Y}(z)$ contains at least $p^{3} d / t(8 s)^{s+1}$ monochromatic edges under $\kappa$. Since every $C_{z}$ occurs, no $\Gamma_{X Y}(z)$ can contain more than $t \cdot 4 s^{2} d_{X Y}(z) / t^{2} \leqslant 16 s^{2} p^{3} d / t$ monochromatic edges under $\kappa$. Therefore

$$
|T| \cdot \frac{16 s^{2} p^{3} d}{t}+(n-|T|) \cdot \frac{p^{3} d}{t(8 s)^{s+1}} \geqslant \frac{p^{3} d n}{4 t(8 s)^{s}} .
$$

From this it follows that $|T|>n /(8 s)^{s+2}$. Since every $C_{z}$ occurs, for every $z \in T, \Gamma_{X Y}(z)$ contains monochromatic edges of at least

$$
\frac{p^{3} d}{t(8 s)^{s+1}} \cdot \frac{t^{2}}{16 s^{2} p^{3} d}>\frac{2 t}{(8 s)^{s+3}}
$$

different colors under $\kappa$. Let $T_{z, \kappa}$ be this set of colors on monochromatic edges of $\Gamma_{X Y}(z)$. We assign random lists to $Z$ of size $s$ from $[t]$. In order for $H^{\prime}$, and therefore $H$, to have 
a list coloring extending $\kappa$, it cannot be that some list at a vertex $z \in T$ is contained in the set $T_{z, \kappa}$. Let $C_{\kappa}$ be the event that no list at any vertex $z \in T$ is contained in $T_{z, \kappa}$. By (4) with $K=(8 s)^{s+3} / 2$,

$$
\left(\begin{array}{c}
2 t /(8 s)^{s+3} \\
s
\end{array}\right)>\frac{1}{(2 K)^{s}}\left(\begin{array}{l}
t \\
s
\end{array}\right)
$$

and therefore

$$
P\left(C_{\kappa}\right)<\left(1-\frac{\left(\begin{array}{c}
2 t /(8 s)^{s+3} \\
s
\end{array}\right)}{\left(\begin{array}{l}
t \\
s
\end{array}\right)}\right)^{|T|}<\exp \left(-\frac{|T|}{(2 K)^{s}}\right)<\exp \left(-\frac{n}{(8 s)^{2} t}\right)
$$

using that $|T|>n /(8 s)^{s+2}$. By $(2), P\left(C_{\kappa}\right)<s^{-3 p n}$, and since there are at most $s^{|X \cup Y|} \leqslant$ $s^{3 p n}$ colorings $\kappa$ of $X \cup Y$, we deduce that there exists a list assignment to the vertices of $Z$ for which no $C_{\kappa}$ occurs. In words, there is an assignment of lists to the vertices of $Z$ such that for any coloring $\kappa$ of $X \cup Y$, some $z \in Z$ cannot be properly colored from its list. For this list assignment, no coloring of $H^{\prime}$ exists. This completes the proof.

\section{$5 \quad$ Note added in proof}

We have learned that the list coloring problem for linear and uniform hypergraphs has been studied independently by Alon and Kostochka [3]. They prove a more general version of Theorem 1, in that they consider $r$-uniform hypergraphs for $r \geqslant 3$, and they do not restrict to $d$-regular hypergraphs but give bounds in terms of the average degree $d$. When specialised to the setting of Theorem 1, their results give similar bounds, and in particular we still do not know whether there exist 3-uniform $d$-regular hypergraphs with list chromatic number $o(\log d)$ as $d \rightarrow \infty$.

\section{Appendix}

We chose $s=(\log d)^{1 / 2}(5 \log \log d)^{-1 / 2}$ and $t=(8 s)^{s(s+4)}$ and put $p:=1 /(8 s)^{3} t$. If $d$ is a large enough constant, we claim that the following three inequalities hold:

$$
\begin{aligned}
t \exp \left(-s^{2} p^{3} d / 4 t^{2}\right) & <1 / 64 d^{2} \\
\exp \left(-n /(8 s)^{2} t\right) & <s^{-3 p n} \\
\exp \left(-p^{2} n / 8\right) & <\exp \left(-n / d^{2}\right) .
\end{aligned}
$$

To verify the inequalities $(6),(7),(8)$ we first observe that with the given definitions we have $s^{2 s^{2}}<d^{1 / 5}$ and so $s^{s^{2}}<d^{1 / 10}$. Thus when $d$ is large enough we find $t=(8 s)^{s(s+4)}<$ $d^{1 / 9}$. Therefore to prove (6) we note that for large enough $d$,

$$
t \exp \left(-s^{2} p^{3} d / 4 t^{2}\right)<d^{1 / 9} \exp \left(-s^{2} d / 4(8 s)^{9} t^{5}\right)<d^{1 / 9} \exp \left(-d^{1 / 3}\right)<1 / 64 d^{2} .
$$

For (7) it suffices to show $3 p \log s<1 / 64 s^{2} t$, which is immediate from the definition of $p$. Finally for (8) we want $1 / d^{2}<p^{2} / 8$, so since $p^{2}=1 /(8 s)^{6} t^{2}>1 / d^{1 / 3}$ the inequality holds provided $d^{5 / 3}>8$. 
Lemma 6 Let $s, t$ be positive integers and suppose that $t / K \geqslant 2 s$. Then

$$
\left(\begin{array}{c}
t / K \\
s
\end{array}\right)>(2 K)^{-s}\left(\begin{array}{l}
t \\
s
\end{array}\right)
$$

Proof $\triangleright$ By definition we have

$$
\left(\begin{array}{c}
t / K \\
s
\end{array}\right)>\frac{(t / K-s)^{s}}{s !}>\frac{1}{s !}\left(\frac{t}{2 K}\right)^{s}
$$

On the other hand

$$
\left(\begin{array}{l}
t \\
s
\end{array}\right) \leqslant \frac{t^{s}}{s !}
$$

and the result follows.

\section{Acknowledgments}

This work was done while the authors were visiting the Institute for Pure and Applied Mathematics at the University of California, Los Angeles. The authors wish to thank IPAM for their support.

\section{References}

[1] Alon, N., Degrees and choice numbers, Random Structures \& Algorithms 16 (2000), 364-368.

[2] Alon, N., Restricted colorings of graphs. In: K. Walker, Editor, Surveys in Combinatorics, Proc 14th British Combinatorial Conference, London Mathematical Society Lecture Notes Series 187, Cambridge Univ. Press (1993), 1-33.

[3] Alon, N., Kostochka, A, Hypergraph list coloring and Euclidean Ramsey theory, submitted, 2010.

[4] Alon, N., Krivelevich, M., The choice number of random bipartite graphs, Annals of Combinatorics 2 (1998), 291-297.

[5] Alon, N., Spencer, J. The Probabilistic Method, Wiley-Interscience, 2000.

[6] Erdös, P., On the combinatorial problems I would most like to see solved, Combinatorica 1 (1981), 25-42.

[7] Erdős, P., On a combinatorial problem. II. Acta Mathematica Academiae Scientiarum Hungaricae 15 (1964), 445-447.

[8] Erdős, P., Lovász, L., Problems and results on 3-chromatic hypergraphs and some related questions, in Infinite and Finite Sets (A. Hajnal et al, eds.), North-Holland, Amsterdam (1975), 609-628. 
[9] Erdős, P., Rubin, A., Taylor, H., Choosability in graphs, Proc. West Coast Conf. on Combinatorics, Graph Theory and Computing, Congressus Numerantium 26 (1979), $125-157$.

[10] Haxell, P., Pei, M., On list coloring Steiner triple systems, Journal of Combinatorial Designs 17 (2009), 314-322.

[11] Kahn, J., Coloring nearly-disjoint hypergraphs with $n+o(n)$ colors, Journal of Combinatorial Theory A 59 (1992), 31-39. 